\title{
Diagnósticos de Trastornos Temporomandibulares en Pacientes Tratados con Radioterapia por Neoplasias Malignas de Cabeza y Cuello: Una Revisión Sistemática Exploratoria de la Literatura
}

\author{
Diagnoses of Temporomandibular Disorders in Patients Treated with Radiotherapy \\ for Malignant Neoplasms of the Head and Neck: Scoping Review
}

\author{
Mario Villegas-Salinas ${ }^{1,2} \&$ Rolando E. Morales-Espinosa ${ }^{3,4}$
}

VILLEGAS-SALINAS, M. \& MORALES-ESPINOSA, R. E. Diagnósticos de trastornos temporomandibulares en pacientes tratados con radioterapia por neoplasias malignas de cabeza y cuello: una revisión sistemática exploratoria de la literatura. Int. J. Odontostomat., 15(3):639-645, 2021.

RESUMEN: Las neoplasias malignas en cabeza y cuello son usualmente tratadas con cirugía combinada con radioterapia en algunos casos, provocando alteraciones en las estructuras maxilofaciales, dejando secuelas en los tejidos relacionados a la articulación temporomandibular (ATM) y la musculatura asociada. La literatura no es clara sobre la evaluación y diagnóstico pretratamiento del paciente oncológico ni su correcta evaluación, por lo que los diagnósticos podrían estar errados. Se realizó una revisión sistemática exploratoria de la literatura presentando la información más actualizada respecto a diagnósticos y alcances relevantes sobre trastornos temporomandibulares (TTMs) en pacientes tratados con radioterapia por cáncer de cabeza y cuello. Se revisaron 4 bases bibliográficas electrónicas incluyendo artículos en inglés originales de enero de 2010 a julio de 2020 enfocados en la evaluación y diagnóstico de TTMs y tejidos asociados morfofuncionalmente en estre grupo de pacientes Se encontraron 353 artículos que aplicados los criterios de inclusión y exclusión se obtuvieron 7 artículos pertinentes para evaluación. La asociación entre el grado de exposición a radioterapia y los cambios moleculares en los tejidos inductores de hipometría mandibular puede ser discutible debido a no considerar un estudio acucioso de la ATM y sus tejidos asociados ni un diagnóstico específico preradioterapia para determinar la preexistencia algún TTM, induciendo su agravamiento o perpetuación. La literatura no es explícita respecto a los signos y síntomas asociados a un correcto examen de la articulación temporomandibular y estructuras asociadas preradioterapia. El trismus es sólo un signo de alteración funcional del sistema estomatognático, pero no un diagnóstico en sí el que debe ser realizado en base a criterios estandarizados y realizado por un Especialista en Dolor Orofacial. Hasta donde sabemos, esta es la primera revisión que intenta resumir la evidencia más actual y relevante sobre los diagnósticos de trastornos temporomandibulares diferentes a "trismus" en este grupo de pacientes.

PALABRAS CLAVE: Cáncer, radioterapia, trastornos temporomandibulares.

\section{INTRODUCCIÓN}

Los cánceres en cabeza y cuello constituyen una de las neoplasias malignas más prevalentes a nivel mundial, con 500000 nuevos casos diagnosticados al año y casi 400000 muertes (Bray et al., 2018; Chen, 2019). Por su ubicación en la ergonomía del macizo facial, la enfermedad puede provocar impacto en acciones tan cotidianas que van desde el acto de comer, hablar o beber, así como también en la interacción con el medio social. El tratamiento aplicado está sujeto a evaluación de las características y

\footnotetext{
${ }^{1}$ Centro de Salud Familiar "Olivar", Olivar, Servicio de Salud O’Higgins, Chile.

${ }^{2}$ Especialista en Trastornos Temporomandibulares y Dolor Orofacial, Unidad de Rehabilitación Bucomaxilofacial, Escuela de Odontología, Universidad de Talca, Talca, Chile.

${ }^{3}$ Cirujano dentista, especialista en Patología y Medicina Oral, Servicio de Odontología, Hospital Gustavo Fricke, Viña del Mar, Chile.

${ }^{4}$ Unidad de Patología y Medicina Oral, Facultad de Odontología, Universidad Andrés Bello, Viña del Mar, Chile.
} 
ubicación del tumor, compromiso loco-regional y definición de su estadiaje, siendo usual la resección radical de la neoplasia combinado en algunos casos con radioterapia, provocando un cambio anatómico de las estructuras maxilofaciales y lesiones en los tejidos blandos con la consecuente impotencia funcional y efectos adversos los que contribuyen de manera negativa en la calidad de vida del paciente (Buglione et al., 2016; van der Geer et al., 2016). Dentro de estas alteraciones se ha documentado las secuelas que deja la radiación sobre los tejidos relacionados a la articulación temporomandibular (ATM) y la musculatura masticatoria asociada, siendo la limitación de la apertura oral (trismus para la literatura en general) el desorden más extensamente registrado producto de la fibrosis de los tejidos a nivel articular y muscular, ocurriendo al cabo de 3 a 6 meses posterior al término de la radioterapia (Buglione et al.; van der Geer et al.), sin embargo también cursan con dolor orofacial inespecífico, mialgias (Romero-Reyes et al., 2015), disfunción oral (Chen) y necrosis de los tejidos blandos (Wu \& Lam, 2016). Los mecanismos biológicos conducentes a trastornos temporomandibulares (TTMs) en el contexto de un paciente oncológico irradiado en cabeza y/o cuello, ya sea de forma directa o indirecta, son de diagnóstico complejo, ya sea por la neoplasia propiamente tal como por el tratamiento (Nicot et al., 2020).

La literatura no entrega claridad respecto a la evaluación y diagnóstico pre-tratamiento del paciente oncológico ni una correcta y acuciosa evaluación de los signos y síntomas previos a nivel de la ATM, por lo que los diagnósticos que se realizan pueden estar errados, malinterpretándose la limitación de la apertura oral como trismus. Tampoco se informa sustancialmente de otros diagnósticos de TTMs asociados a pacientes irradiados por cáncer de cabeza y cuello.

El objetivo del presente artículo es presentar la información más actualizada respecto a diagnósticos de TTMs en pacientes tratados con radioterapia por neoplasias malignas a nivel de cabeza y cuello y los alcances destacados de las investigaciones al respecto, además de definir y proyectar el examen de la ATM como una herramienta rutinaria en pacientes oncológicos pre y post tratamiento.

\section{MATERIAL Y MÉTODO}

Se realizó un estudio descriptivo a través de una revisión sistemática exploratoria (scoping review) ba- sado en los criterios propuestos por la guía PRISMAScR (Tricco et al., 2018), respondiendo a la pregunta orientativa estructurada: ¿Cuál es la evidencia más reciente sobre diagnósticos de TTMs en pacientes con cáncer de cabeza y cuello que recibieron radioterapia?. Para identificar artículos potencialmente relevantes para el estudio, se revisaron 4 bases bibliográficas: PubMed/MEDLINE, SciVerse Scopus, ISI Web of Science y ScienceDirect. Se incluyeron aquellos artículos en inglés originales, tanto publicados electrónicamente así como aquellos manuscritos aceptados pero no publicados, documentados en el periodo de enero de 2010 a julio de 2020 y cuyo tema de estudio sea la evaluación de alteraciones a nivel de la ATM y tejidos asociados morfofuncionalmente al sistema estomatognático y diagnósticos de TTM, todo en pacientes con cáncer de cabeza y cuello sometidos a radioterapia. Se incluyeron los diseños de estudios de casos y controles, series de casos y cohortes (retrospectivos/prospectivos). Se excluyen artículos repetidos entre bases bibliográficas, resúmenes sin disponibilidad de texto completo, publicaciones a texto completo no disponibles en formato electrónico, revisiones bibliográficas y publicaciones no científicas como: cartas al editor, noticias, indicadores, reseñas bibliográficas, memorias o resúmenes de congresos, carteles, guías, trípticos y capítulos de libros. Para la búsqueda de artículos se seleccionaron aquellas investigaciones que en su título o resumen deben contener uno o más de los términos claves seleccionados y considerados relevantes tanto individualmente como su combinación en los algoritmos de búsqueda: "[irradiated] AND [patients] AND [TMJ disorders] AND [oncology]"; "[head and neck] AND [radiation] AND [temporomandibular disorders]"; "[temporomandibular disorders] AND [head and neck] AND [cancer] AND [radiotherapy]" y "[radio-induced] AND [temporomandibular disorders]" los que fueron numerados e ingresados a los buscadores generales de temas de cada base de datos con el filtro de "TODOS LOS CAMPOS" disponible en cada sitio o su equivalente a criterio de uno de los investigadores (RM), tabulando en una matriz bibliométrica general los detalles sobre los datos bibliográficos relevantes para la búsqueda del artículo a texto completo. Los artículos disponibles en formato a Full-text fueron leídos independientemente por ambos autores (MV y RM), llegando a consenso mutuo para la selección cualitativa final, en concordancia con el objetivo del trabajo y los criterios de inclusión y exclusión. Finalmente, en una matriz de resumen general se registró la información pertinente para cada artículo seleccionado, resumiendo las variables de interés. 
VILLEGAS-SALINAS, M. \& MORALES-ESPINOSA, R. E. Diagnósticos de trastornos temporomandibulares en pacientes tratados con radioterapia por neoplasias malignas de cabeza y cuello: una revisión sistemática exploratoria de la literatura. Int. J. Odontostomat., 15(3):639-645, 2021.

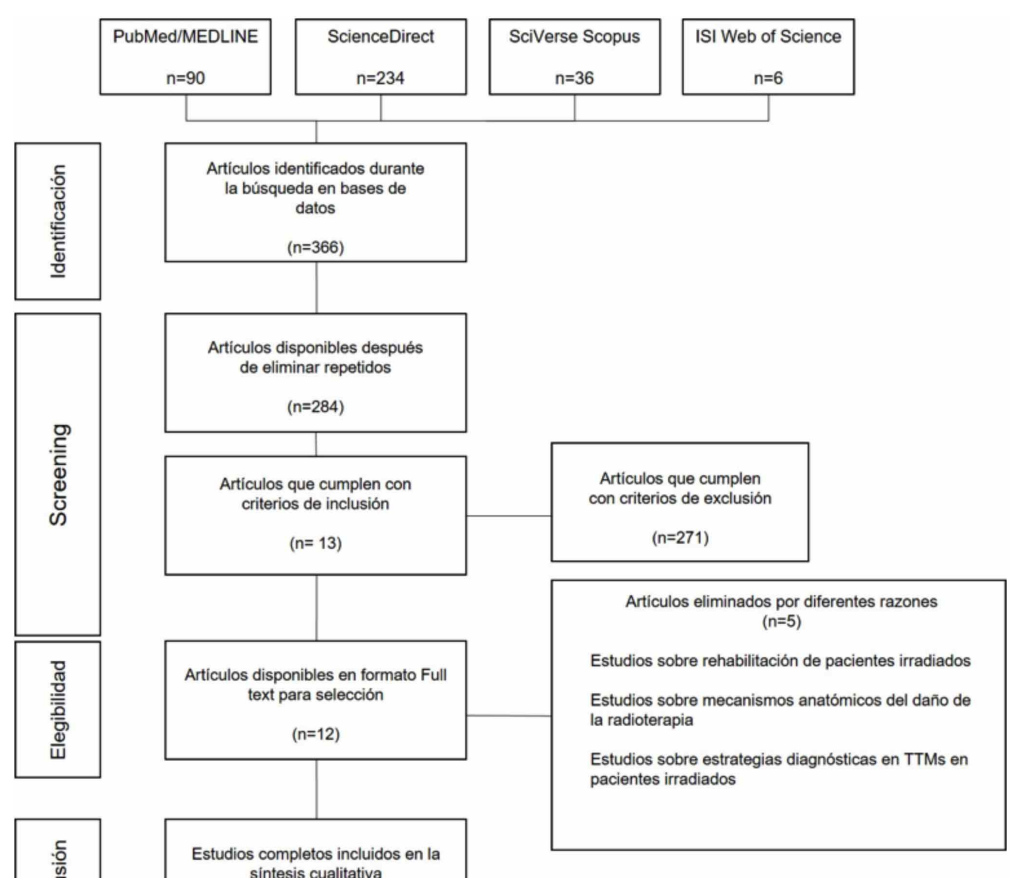

Fig. 1. Flujograma PRISMA de la búsqueda bibliográfica.

\section{RESULTADOS}

Se encontraron 353 artículos de los cuales una vez eliminados los repetidos y aplicados los criterios de inclusión y exclusión se obtienen 7 artículos pertinentes a formato completo para evaluación. El resumen del proceso y los resultados de la búsqueda se grafican en el flujograma PRISMA (Fig. 1).

Los artículos incluidos en esta revisión de la literatura fueron publicados entre los años 2011 a 2019 y son de 7 diferentes países, distribuyéndose en 2 europeos, 1 americano, 3 asiáticos y 1 africano. En términos de las disciplinas de las ciencias de la salud se identificaron 1 de cirugía oral y maxilofacial, 1 de dolor orofacial, 2 de oncología y 3 de multidisciplinarios. La información general y las características de los artículos incluidos, junto a los hallazgos más relevantes están descritos en la Tabla I.

Tabla I. Matriz de resumen general con los principales hallazgos de los estudios incluidos.

\begin{tabular}{|c|c|c|c|}
\hline Autor (año) & País & $\mathrm{n}$ & TTMs reportados/Principales hallazgos \\
\hline Chen et al. (2011) & Taiwan & $\begin{array}{l}H=71 \\
M=1\end{array}$ & $\begin{array}{ll}\text { - } & \text { Dolor espontáneo y en función con restricción } \\
\text { - } & \text { Dolor en función fue mayor que dolor espontáneo en T1 y T2 } \\
\text { - } & \text { Dificultad para comer mayor en T2 } \\
\text { - Sensibilidad al tacto posiblemente relacionada a dolor neuropático }\end{array}$ \\
\hline Mercado et al. (2014) & EEUU & $\begin{array}{l}H=2 \\
M=1\end{array}$ & $\begin{array}{l}\text { - Erosión y pérdida de volumen condilar } \\
\text { - } \quad \text { Dolor articular y disminución de ROM } \\
\text { - Asimetría facial }\end{array}$ \\
\hline Agarwal et al. (2016) & India & $\begin{array}{l}\mathrm{H}=18 \\
\mathrm{M}=12\end{array}$ & $\begin{array}{l}\text { - } \quad \text { Metástasis linfonodal positiva puede predecir la apertura oral preradioterapia } \\
\text { - } \quad \text { Estadios avanzados de la enfermedad tienen mayor prevalencia de trismus } \\
\text { - } \quad \text { Mayor prevalencia de trismus en maxibulectomizados } \\
\text { - } \quad \text { La hay reducción significativa en la apertura oral al comparar post RT y postcirugía } \\
\text { - } \quad \text { encia al tto. del trismus en estos pacientes }(p=0,02)\end{array}$ \\
\hline Galitis et al. (2017) & Grecia & $\begin{array}{l}H=9 \\
M=1\end{array}$ & $\begin{array}{l}\text { - } \quad 37,5 \% \text { tenían apertura oral reducida y menor funcionalidad de la ATM } \\
\text { - } \quad 2 / 10 \text { pacientes tenían sonidos y clicks } \\
\text { - } \quad 6 / 10 \text { mialgias localizadas } \\
\text { - } \quad 1 / 10 \text { anomalía funcional severa } \\
\text { - } \quad 2 / 7 \text { pacientes con dolor de cabeza } \\
\text { - } \quad 6 \text { pacientes con mucositis oral grado III }\end{array}$ \\
\hline Wu et al. (2017) & China & $\begin{array}{l}H=15 \\
M=24\end{array}$ & $\begin{array}{ll}\text { - } & \text { Incidencia de trismus } 35 \% \\
\text { - } & \text { Disco articular: } 11 \% \text { más delgado que grupo control } \\
\text { - } & \text { Reducción de ROM: } 26 \% \text { más que grupo control } \\
\text { - } & \text { Irregularidad condilar mayor en grupo de pacientes } \\
\end{array}$ \\
\hline Elgohary et al. (2018) & Egipto & $\mathrm{H}=33 \quad \mathrm{M}=27$ & $\begin{array}{l}-\quad \text { EVA Grupo A 7.75 } \pm 1.41, \text { GRUPO B 7.55 } \pm 1.61, \text { GRUPO C } 7.70 \pm 1.08 \\
\text { - } \quad \text { ROM Grupo A } 25.50 \pm 6.77068 \text {, Grupo B } 23.65 \pm 5.42193 \text {, Grupo C } 24.10 \pm 4.95134\end{array}$ \\
\hline Pauli et al.(2019) & Suecia & $\begin{array}{l}\mathrm{H}=57 \\
\mathrm{M}=32\end{array}$ & $\begin{array}{l}\text { - } \quad \text { Alta incidencia de TTM en evaluación inicial } 68 \% \\
\text { - } \quad 33 \% \text { con apertura oral reducida } \\
\text { - } \quad \text { Aumento sensibilidad muscular masetero y temporal en su inserción } \\
\text { - } \quad \text { Signos de artralgia y mialgia } \\
\text { - } \quad \text { Temporal anterior menos afectado } \\
\text { - } \quad \text { ATM con poca sensibilidad a la palpación } \\
\text { - } \quad \text { No se reportó aumento de dolor facial } \\
\text { - } 94 \% \text { de los pacientes a los } 6 \text { meses tenían signos objetivos y subjetivos de TTM }\end{array}$ \\
\hline
\end{tabular}




\section{DISCUSIÓN}

La revisión narrativa exploratoria presentada resume la información más actualizada respecto a diagnósticos referidos a TTM en pacientes sometidos a radioterapia por cáncer de cabeza y cuello, destacando la relevancia que otorgan los artículos a la presencia de trastornos articulares de la ATM y mialgias de los músculos masticatorios, relacionando dichas alteraciones con la pérdida de función y dolor en el sistema estomatognático en este grupo particular de pacientes.

Radioterapia y TTM. La radioterapia genera en las estructuras orofaciales daño celular directo, pérdida de perfusión vascular y estimulación de cambios tendientes a la fibrosis (Toljanic et al., 2002). El sistema musculoesquelético se ve afectado por estos cambios, siendo el signo más evidente la disminución del rango de movimiento mandibular que disminuye en promedio de $26 \%$ a un $37 \%$ en comparación con el rango de movimiento detectado antes de que los tejidos sean expuestos a la radiación (Galitis et al., 2017; Wu et al., 2017; Pauli et al., 2019). El grado de daño es directamente proporcional a la dosis de radiación en los tejidos, por lo que se entiende que mientras más cerca de la ATM y los músculos masticatorios sea dirigida, mayor compromiso funcional y estructural de estos tejidos, observado en tumores alojados en la glándula parótida y en oro/nasofaringe (Gebre-Medhin et al., 2016).

Alteraciones Musculares. Los estudios incluidos en la revisión reportan que la sensibilidad y dolor en los músculos involucrados en el cierre mandibular fueron los que presentaron mayor sintomatología post radioterapia, siendo el músculo masétero el más recurrente a la hora de identificar algún trastorno muscular (Galitis et al.; Pauli et al.). Esto se condice con reportes anteriores donde se menciona la pérdida de función de este debido a la gran carga de radiación a la que es expuesto durante la terapia. En un seguimiento a 6 meses post radioterapia un $53,9 \%$ pacientes presentan sensibilidad bilateral en el músculo masetero, y a los 12 meses un 40,2 \% seguía con la misma sintomatología (Pauli et al.).

En cuanto a las metodologías utilizadas para la pesquisa de mialgias en el territorio orofacial la literatura consultada no especifica cuál fue el protocolo utilizado en la evaluación de la musculatura masticatoria, lo que conlleva a la ausencia de diagnósticos mus- culares específicos tales como co-contracción protectora, dolor miofascial, dolor muscular local, mialgia de mediación central, miositis, mioespasmo, entre otros (Okeson, 2013; De Lew 2018). Lo anterior puede ser explicado por la ausencia de un especialista en el área de dolor orofacial en el desarrollo de las investigaciones y la ausencia de criterios diagnósticos empleados en base a algún sistema estandarizado como el DC/TMD (Schiffman et al., 2014), que cuenta con un protocolo validado para la realización de un examen muscular mediante la aplicación de $1 \mathrm{~kg}$ de fuerza en músculos extraorales, y así determinar sus diagnósticos posibles en base a la sintomatología encontrada en los pacientes evaluados.

Alteraciones Articulares. La literatura muestra que existen variadas presentaciones de este tipo de alteraciones relacionadas con el funcionamiento de la articulación temporomandibular. Una de ellas es la presencia de artralgia tanto para la masticación como en la ejecución de otras actividades como por ejemplo hablar, tragar o morder (Chen et al., 2011; Galitis et al.; Elgohary et al., 2018). Esto puede ser explicado por la presencia de inflamación de los tejidos sinoviales de la articulación y los cambios biológicos generados como el daño celular, pérdida de irrigación tisular y cambios fibróticos que se producen por la exposición a la radiación, reduciendo la dinámica articular con la subsecuente disminución del rango de apertura bucal progresiva en el tiempo, con mayor dolor e impotencia funcional (Rapidis et al., 2015; List \& Jensen, 2017).

Con respecto a los trastornos internos de la articulación de tipo estructurales, sólo un estudio menciona la presencia de ruidos articulares posterior al tratamiento por radioterapia (Pauli et al.). Por lo tanto, diagnósticos comunes en la población general como luxaciones discales con o sin reducción, dolorosas o no y que pueden generar hipometría mandibular, no son considerados como posibles factores en el desarrollo o perpetuación de una disminución del movimiento articular. Por otro lado, el curso natural de los desplazamientos discales con reducción es variable en el tiempo y no se indica en los estudios si su presencia es anterior a la exposición a la radiación (Agarwal et al., 2016; Poluha et al., 2019).

En relación a los estudios presentes en esta revisión, se reportaron cambios a nivel óseo en la ATM al ser sometida a dosis de radiación directa. Se mencionan erosiones, irregularidades y pérdidas de volumen condilar tras el tratamiento con radioterapia, pudiendo incluso generar alteraciones en la simetría fa- 
cial (Mercado et al., 2014; Wu et al.). Estos cambios óseos pueden ser explicados por el aumento del metabolismo que se produce en los tejidos sinoviales en combinación con el daño celular directo. Algunos de los cambios degenerativos que se han observado son el engrosamiento del líquido sinovial y adelgazamiento del cartílago articular (Bensadoun et al., 2010; Rapidis et al.).

La metodología de los estudios reporta presencia de dolor articular, pero no se especifica qué zona articular es la dolorosa, por ende, no se sabe si son los tejidos sinoviales laterales o retrodiscales que están sintomáticos en el momento del examen. Un defecto de estos estudios es llegar a conclusiones de dolor articular en base a cuestionarios dirigidos a pacientes y no complementarlo con un examen físico exhaustivo de la articulación. Por lo que se sugiere la aplicación del examen articular propuesto por DC/TMD en donde se realiza una palpación calibrada del polo lateral de $0,5 \mathrm{~kg}$ para determinar la presencia de dolor, localizarlo de mejor forma y finalmente tener un diagnóstico más certero.

Por otra parte, si bien se describen cambios óseos, no se determina nuevamente si estamos en presencia de una osteoartritis de ATM o algún otro trastorno que disminuya el volumen condilar o genere cambios a nivel del cartílago articular, es decir, la presencia de alguna patología degenerativa secundaria a la radioterapia. En relación a esto, los estudios en general no emplean buenas herramientas imagenológicas para llegar a un diagnóstico acabado, puesto que muy pocos utilizan RNM (Resonancia Nuclear Magnética) o CBCT (Cone Beam Computer Tomography) para graduar la presencia de cambios óseos y de tejido blando a nivel articular. Se sugiere que para estandarizar los cambios óseos que se desarrollan en la ATM posteriores a radioterapia debería realizarse $2 \mathrm{CBCT}$ : uno inicial y así determinar efectivamente la presencia de una patología degenerativa ósea, y otro posterior al tratamiento con radioterapia, para evidenciar si el proceso patológico fue acelerado o sólo es parte del curso natural de la enfermedad.

Funcionalidad. El signo más prevalente en los pacientes oncológicos de cabeza y cuello sometidos a radioterapia es la hipometría o reducción del rango de apertura bucal, seguido por la presencia de dolor o sensibilidad en la musculatura. En la literatura se encuentra ampliamente descrito un término que engloba cualquier disminución de la apertura oral, pero sin especificar cuáles son los diagnósticos específicos que llevan a ese estado musculoesquelético. El término es conocido como "trismus", el cual se define como la presencia de una apertura bucal menor igual a $35 \mathrm{~mm}$ (van der Geer et al.). Si bien nos proporciona información sobre la pérdida de funcionalidad en cuanto a la artrodinamia mandibular, conceptualmente es sólo un signo de alguna alteración funcional del sistema estomatognático, pero no un diagnóstico en sí. De hecho, en ninguna de las clasificaciones más utilizadas en la actualidad sobre Trastornos Temporomandibulares, en donde podemos mencionar la de AAOP o DC/TMD (Schiffman et al.; Ohrbach \& Dworkin, 2016), aparece el diagnóstico de trismus siendo una inquietud que hasta estos días se siga ocupando ése término para evaluar la situación de pérdida de funcionalidad en pacientes sometidos a tratamiento de radioterapia sin tener en cuenta diagnósticos específicos de TTM. Lo anterior, porque actualmente existen criterios diagnósticos establecidos internacionalmente para realizar estudios científicos sobre los trastornos temporomandibulares. EI DC/ TMD proporciona un protocolo estandarizado (Schiffman et al.) para determinar la presencia de patologías presentes en el sistema musculoesquelético orofacial, y por ende debería ser el instrumento indicado, puesto que cuenta con tópicos como la localización del dolor, funcionalidad de la ATM, rango de apertura bucal, presencia de ruidos articulares, palpación muscular y articular, y finalmente un ítem de diagnóstico. De ahí su importancia cuando queremos obtener datos sobre presencia de trastornos en el sistema y a la vez, ver cómo cambian en el tiempo tras una terapia como la radioterapia en pacientes con cáncer de cabeza y cuello.

\section{CONCLUSIÓN}

La asociación que comúnmente se hace entre el grado de exposición a radioterapia y la generación de cambios moleculares y fibróticos en los tejidos como ecuación principal del desarrollo de un hipometría mandibular puede ser discutible e incompleta ya que varios de los estudios no consideran la práctica de un estudio acucioso de la ATM y sus tejidos asociados con un diagnóstico específico pre-radioterapia para determinar la presencia de algún TTM previo al tratamiento oncológico, y que dado a que no existe la certeza del diagnóstico inicial del paciente, puede que un porcentaje no menor de pacientes presenten hipometría mandibular o algún trastorno articular/muscular previo que determine finalmente un agravamiento de la hipomovilidad articular o perpetuación del cuadro post-radioterapia. 
Los estudios clínicos en ese sentido deberían tener un dato diagnóstico inicial y otro posterior al tratamiento de este grupo de pacientes de modo de visualizar si los cambios generados en el sistema son netamente producidos por la radioterapia o por combinación de patologías previas con la radioterapia.

Hasta donde sabemos, esta es la primera revisión de alcance que intenta resumir la evidencia más actual y relevante sobre los diagnósticos de trastornos temporomandibulares en pacientes tratados con radioterapia por neoplasias malignas de cabeza y cueIlo. Como pudimos observar durante la realización de la revisión, la literatura no ha sido suficientemente explícita respecto a los signos y síntomas asociados a un correcto examen de la articulación temporomandibular, musculatura masticatoria y estructuras asociadas pre-radioterapia. Es por esto que el diagnóstico en base a criterios estandarizados y realizado por un Especialista en Dolor Orofacial idealmente, propone al equipo médico un nuevo desafío en la pesquisa de alteraciones funcionales relacionadas al sistema estomatognático, lo cual es relevante en el contexto del tratamiento y rehabilitación de pacientes oncológicos tratados con radioterapia.

VILLEGAS-SALINAS, M. \& MORALES-ESPINOSA, R. E. Diagnoses of temporomandibular disorders in patients treated with radiotherapy for malignant neoplasms of the head and neck: Scoping review. Int. J. Odontostomat., 15(3):639-645, 2021.

ABSTRACT: Head and neck malignant neoplasms are usually treated with surgery combined with radiotherapy, causing alterations in the maxillofacial structures, leaving sequelae in the tissues related to the temporomandibular joint (TMJ) and associated muscles. The literature is not clear about the pretreatment evaluation and diagnosis of cancer patients or their correct evaluation, so the diagnoses could be wrong. An scoping review of the literature was carried out presenting the most up-to-date information regarding the diagnoses and relevant outcomes of temporomandibular disorders (TMDs) in patients treated with radiotherapy for head and neck cancer. Four (4) electronic bibliographic databases were reviewed including original articles in English from January 2010 to July 2020 focused on the evaluation and diagnosis of TTMs and morphofunctionally associated tissues in a narrow group of patients. 353 articles were found that applied the inclusion and exclusion criteria. 7 relevant articles for evaluation. The association between the degree of exposure to radiotherapy and the molecular changes in the tissues that induce mandibular hypometry may be debatable due to not considering a careful study of the TMJ and its associated tissues, nor a specific pre-radiotherapy diagnosis to determine the preexistence of some TMD, inducing its aggravation or perpetuation. The literature is not explicit regarding the signs and symptoms associated with a correct examination of the temporomandibular joint and associated structures prior to radiotherapy. Trismus is only a sign of functional alteration of the stomatognathic system, but not a diagnosis in itself, which must be made based on standardized criteria and performed by an Orofacial Pain Specialist. To our knowledge, this is the first review that attempts to summarize the most current and relevant evidence on the diagnoses of temporomandibular disorders other than "trismus" in this group of patients.

KEY WORDS: cancer, radiotherapy, temporomandibular disorders.

\section{REFERENCIAS BIBLIOGRÁFICAS}

Agarwal, P.; Shiva Kumar, H. R. \& Rai, K. K. Trismus in oral cancer patients undergoing surgery and radiotherapy. J. Oral Biol. Craniofac. Res., 6(Suppl. 1):S9-S13, 2016.

Bensadoun, R. J.; Riesenbeck, D.; Lockhart, P. B.; Elting, L. S.; Spijkervet, F. K. L.; Brennan, M. T. \& Trismus Section, Oral Care Study Group, Multinational Association for Supportive Care in Cancer (MASCC)/International Society of Oral Oncology (ISOO). A systematic review of trismus induced by cancer therapies in head and neck cancer patients. Support. Care Cancer, 18(8):1033-8, 2010.

Bray, F.; Ferlay, J.; Soerjomataram, I.; Siegel, R. L.; Torre, L. A. \& Jemal, A. Global cancer statistics 2018: GLOBOCAN estimates of incidence and mortality worldwide for 36 cancers in 185 countries. CA Cancer J. Clin., 68(6):394-424, 2018.

Buglione, M.; Cavagnini, R.; Di Rosario, F.; Maddalo, M.; Vassalli, L.; Grisanti, S.; Salgarello, S.; Orlandi, E.; Bossi, P.; Majorana, A.; et al. Oral toxicity management in head and neck cancer patients treated with chemotherapy and radiation: Xerostomia and trismus (Part 2). Literature review and consensus statement. Crit. Rev. Oncol. Hematol., 102:47-54, 2016.

Chen, S. C. Oral dysfunction in patients with head and neck cancer: a systematic review. J. Nurs. Res., 27(6):e58, 2019.

Chen, S. C.; Liao, C. T. \& Chang, J. T. C. Orofacial pain and predictors in oral squamous cell carcinoma patients receiving treatment. Oral Oncol., 47(2):131-5, 2011.

Elgohary, H. M.; Eladl, H. M.; Soliman, A. H. \& Soliman, E. S. Effects of ultrasound, laser and exercises on temporomandibular joint pain and trismus following head and neck cancer. Ann. Rehabil. Med., 42(6):846-53, 2018.

Galitis, E.; Droukas, V.; Tzakis, M.; Psarras, V.; Galiti, D.; Kyrodimos, E.; Trichas, M.; Psyrri, A.; Papadogeorgakis, N.; Kouri, M.; et al. Trismus and reduced quality of life in patients with oral squamous cell carcinoma, who received post-operative radiotherapy alone or combined with chemotherapy. Forum Clin. Oncol., 8(1):29-36, 2017.

Gebre-Medhin, M.; Haghanegi, M.; Robért, L.; Kjellén, E. \& Nilsson, $\mathrm{P}$. Dose-volume analysis of radiation-induced trismus in head and neck cancer patients. Acta Oncol., 55(11):1313-7, 2016.

List, T. \& Jensen, R. H. Temporomandibular disorders: Old ideas and new concepts. Cephalalgia, 37(7):692-704, 2017.

Nicot, R.; Raoul, G.; Ferri, J. \& Schlund, M. Temporomandibular disorders in head and neck cancers: Overview of specific mechanisms and management. J. Stomatol. Oral Maxillofac. Surg., 121(5):563-8, 2020. 
VILLEGAS-SALINAS, M. \& MORALES-ESPINOSA, R. E. Diagnósticos de trastornos temporomandibulares en pacientes tratados con radioterapia por neoplasias malignas de cabeza y cuello: una revisión sistemática exploratoria de la literatura. Int. J. Odontostomat., 15(3):639-645, 2021.

Ohrbach, R. \& Dworkin, S. F. The evolution of TMD diagnosis: past, present, future. J. Dent. Res., 95(10):1093-101. 2016.

Pauli, N.; Mejersjö, C.; Fagerberg-Mohlin, B. \& Finizia, C. Temporomandibular disorder in head and neck cancer patients undergoing radiotherapy: Clinical findings and patient-reported symptoms. Head Neck, 41(10):3570-6, 2019.

Poluha, R. L.; De La Torre Canales, G.; Costa, Y. M.; Grossmann, E.; Bonjardim, L. R. \& Conti, P. C. R. (2019). Temporomandibular joint disc displacement with reduction: A review of mechanisms and clinical presentation. J. Appl. Oral Sci., 27:e20180433, 2019.

Rapidis, A. D.; Dijkstra, P. U.; Roodenburg, J. L. N.; Rodrigo, J. P.; Rinaldo, A.; Strojan, P.; Takes, R. P. \& Ferlito, A. Trismus in patients with head and neck cancer: Etiopathogenesis, diagnosis and management. Clin. Otolaryngol., 40(6):516-26, 2015.

Romero-Reyes, M.; Teruel, A. \& Ye, Y. Cancer and Referred Facial Pain. Curr. Pain Headache Rep., 19(8):37, 2015.

Schiffman, E.; Ohrbach, R.; Truelove, E.; Look, J.; Anderson, G.; Goulet, J. P.; List, T.; Svensson, P.; Gonzalez, Y.; Lobbezoo, F.; et al. Diagnostic Criteria for Temporomandibular Disorders (DC/ TMD) for Clinical and Research Applications: recommendations of the International RDC/TMD Consortium Network* and Orofacial Pain Special Interest Groupt. J. Oral Facial Pain Headache, 28(1):6-27, 2014.

Toljanic, J. A.; Heshmati, R. H. \& Bedard, J. F. Dental follow-up compliance in a population of irradiated head and neck cancer patients. Oral Surg. Oral Med. Oral Pathol. Oral Radiol. Endod., 93(1):35-8, 2002.

Tricco, A. C.; Lillie, E.; Zarin, W.; O’Brien, K. K.; Colquhoun, H.; Levac, D.; Moher, D.; Peters, M. D. J.; Horsley, T.; Weeks, L.; et al. PRISMA extension for scoping reviews (PRISMA-ScR): Checklist and explanation. Ann. Int. Med., 169(7):467-73, 2018.

van der Geer, S. J.; Kamstra, J. I.; Roodenburg, J. L. N.; van Leeuwen, M.; Reintsema, H.; Langendijk, J. A. \& Dijkstra, P. U. Predictors for trismus in patients receiving radiotherapy. Acta Oncol., 55(11):1318-23, 2016.

Wu, V. W. C. \& Lam, Y. N. Radiation-induced temporo-mandibular joint disorder in post-radiotherapy nasopharyngeal carcinoma patients: assessment and treatment. J. Med. Radiat. Sci., 63(2):124-32, 2016

Wu, V. W. C.; Ying, M. T. C. \& Kwong, D. L. W. A study on the postradiotherapy changes of temporomandibular joint in nasopharyngeal carcinoma patients. Br. J. Radiol., 90(1080):20170375, 2017.

\author{
Dirección para correspondencia: \\ Rolando E. Morales Espinosa \\ Unidad de Patología y Medicina Oral \\ Facultad de Odontología \\ Universidad Andrés Bello \\ Calle Quillota \#980, Torre E, Segundo Piso \\ Teléfono: $56-32-2845108$ \\ Viña del Mar \\ CHILE
}

\section{CHILE}

E-mail: rmoralesespinosa@uandresbello.edu 